\title{
Optimal weighting method for interval-valued intuitionistic fuzzy opinions
}

\author{
M. El Alaoui ${ }^{1}$, H. Ben-Azza ${ }^{1}$ and K. El Yassini ${ }^{2}$ \\ ${ }^{1}$ Department of Production and Industrial Engineering \\ Moulay Ismail University, Meknes, Morocco \\ e-mails: mohamedelalaoui208@gmail.com, \\ hbenazza@yahoo.com \\ ${ }^{2}$ IA Laboratory, Faculty of Sciences \\ Moulay Ismail University, Meknes, Morocco \\ e-mail: Khalid.ElYassini@gmail.com
}

Received: 28 March 2018

Revised: 7 June 2018

Accepted: 26 October 2018

\begin{abstract}
In this work, we propose a method to achieve consensus in a group decision making situation, where the opinions are described by interval-valued intuitionistic fuzzy sets. Optimality is achieved by minimizing weighed incoherencies. An illustrative example is proposed.
\end{abstract}

Keywords: Optimal weighing, Intuitionistic fuzzy set, Interval-valued intuitionistic fuzzy set. 2010 Mathematics Subject Classification: 03E72, 90B50.

\section{Introduction}

Since unanimity is rarely achieved in group decision making, a certain level of consensus might be acceptable. The achieved consensus must take into consideration human uncertainty, to do so, we model the expressed opinions by interval-valued intuitionistic fuzzy numbers. In the rest of this manuscript the needed background for fuzzy logic is presented in Section 2, while Section 3 encompasses the used algorithm with an illustrative example. 


\section{Preliminaries}

In classical sets, each element either belongs to a certain set or not at all, while in fuzzy set theory a certain degree of membership is tolerated [13]. Let $X$ be a set and $F$ be a fuzzy set in $X$,where $F$ is defined as follows:

$$
F=\left\{\left\langle x, \mu_{F}(x)\right\rangle \mid x \in X\right\},
$$

where $\mu_{F}(x)$ is the degree of membership of $x$ in $F$ in the unity interval:

$$
\mu_{F}: X \longrightarrow[0,1] \text {. }
$$

Atanassov [1, 2] extended the notion of fuzzy sets to intuitionistic fuzzy sets (IFS). An intuitionistic fuzzy set $A$ is defined as follows:

$$
A=\left\{\left\langle x, \mu_{A}(x), \nu_{A}(x)\right\rangle \mid x \in X\right\},
$$

where $\mu_{A}(x)$ and $\nu_{A}(x)$ are respectively the membership function and the non-membership function, with the following conditions:

$$
\begin{gathered}
\mu_{A}: X \longrightarrow[0,1], \nu_{A}: X \longrightarrow[0,1] \\
\mu_{A}(x)+\nu_{A}(x) \leq 1 \quad \forall x \in X .
\end{gathered}
$$

The hesitancy function can be computed by the following formula:

$$
\pi_{A}(x)=1-\left[\mu_{A}(x)+\nu_{A}(x)\right] \quad \forall x \in X .
$$

The fuzzy sets were presented in order to permit human uncertainty, while it is counterintuitive to demand an exact membership function and non-membership function. In that sense Atanassov and Gargov [4] extended the IFS to interval-valued intuitionistic fuzzy sets (IVIFS) fulfilling the following:

$$
A=\left\{\left\langle x, M_{A}(x), N_{A}(x)\right\rangle \mid x \in X\right\},
$$

where $M_{A}(x) \subset[0,1]$ and $N_{A}(x) \subset[0,1]$ are respectively the membership interval and the non-membership interval, and for these two intervals it holds that [4]:

$$
\sup M_{A}(x)+\sup N_{A}(x) \leq 1 .
$$

For convenience, we note an interval-valued fuzzy number as $\beta=([a, b],[c, d])$ where $a=$ $\inf M_{\beta}, b=\sup M_{\beta}, c=\inf N_{\beta}$ and $d=\sup N_{\beta}$ are interval numbers.

Let $\beta_{i}=\left(\left[a_{\beta_{i}}, b_{\beta_{i}}\right],\left[c_{\beta_{i}}, d_{\beta_{i}}\right]\right)$ be a collection of interval-valued intuitionistic fuzzy numbers, the main aggregation operators are the interval-valued intuitionistic fuzzy weighting averaging $I I F W A$, and the interval-valued intuitionistic fuzzy weighting geometric IIFWG [11], hence the aggregated value according to IIFWA is:

$$
\operatorname{IIFW} A_{w}\left(\beta_{1}, \beta_{2}, \ldots, \beta_{n}\right)=([a, b],[c, d]),
$$

where

$$
a=1-\prod_{i=1}^{n}\left(1-a_{\beta_{i}}\right), \quad b=1-\prod_{i=1}^{n}\left(1-b_{\beta_{i}}\right), \quad c=1-\prod_{i=1}^{n} c_{\beta_{i}}, \quad d=1-\prod_{i=1}^{n} d_{\beta_{i}}
$$

and $w_{i}$ are the weights of the respective $\beta_{i}$.

The main question is how to attribute the correct weight to each decision. 


\section{Proposed method}

Several method exists in the literature to attribute the correct weights $[5,7,8,12,14]$. Here we propose to follow the procedure proposed in [7] to the IVIFS. The desired consensus is achieved by minimizing the following function:

$$
\min _{M \times \mathbb{R}^{4}} \sum_{i=1}^{n} w_{i}^{m} *\left(c-S\left(\beta_{i}, \beta\right)\right),
$$

where $M=\left\{\begin{array}{c}W=\left(w_{1}, w_{2}, \ldots, w_{n}\right), w_{i} \geq 0 \\ \sum_{i=1}^{n} w_{i}=1\end{array}\right\}, m$ is a positive integer $(m>1), S\left(\beta_{i}, \beta\right)$ is the similarity between the $i$-th decision and the consensus, $c$ is a real number $(c>1)$.

Several methods have been proposed to compute similarity from a distance $[6,9,10]$, here we adopt the Hamming distance for IVIFS [3], and derive the similarity as by Santini and Jain [9] to ease computation $S=1-D$. Hence, the distance between two IVIFS $\beta_{1}$ and $\beta_{2}$ is:

$$
D\left(\beta_{1}, \beta_{2}\right)=\frac{1}{2}\left(\left|a_{1}-a_{2}\right|+\left|b_{1}-b_{2}\right|+\left|c_{1}-c_{2}\right|+\left|d_{1}-d_{2}\right|\right) .
$$

\subsection{Algorithm}

Step 1: Each expert $E_{i}: 1 \leq i \leq n$ assesses each alternative using an IVIFS.

Step 2: Set the initial aggregation weights such that $0 \leq w_{i}^{(0)} \leq 1$ and $\sum_{i=1}^{n} w_{i}=1$. The iterations are labeled $l=0,2, \ldots$

Step 3: Compute the aggregated consensus at Step $l$ :

$$
\beta^{l}=I I F W A\left(\beta_{i}\right)
$$

Step 4: Let $W^{l}=\left(w_{1}^{(l)}, w_{2}^{(l)}, \ldots, w_{n}^{(l)}\right)$. Compute $W^{l+1}$ as follows :

$$
W^{l+1}=\frac{\left(1 /\left(c-S\left(\beta^{l}, \beta_{i}\right)\right)\right)^{1 /(m-1)}}{\sum_{j=1}^{n}\left(1 /\left(c-S\left(\left(\beta^{l}, \beta_{i}\right)\right)\right)^{1 /(m-1)}\right.} .
$$

Step 5: If $\left\|W^{l+1}-W^{l}\right\|>\varepsilon$, set $l=l+1$ and go to Step 3. Else Stop.

\subsection{Illustrative example}

Let three experts assess an alternative as follows: $\beta_{1}=([0.22,0.31] ;[0.23,0.54]), \beta_{2}=$ $([0.04,0.21] ;[0.35,0.46])$ and $\beta_{3}=([0.25,0.27] ;[0.23,0.4])$.

We choose $m=2, c=1.5$ and $W^{0}=(1,0,0)$. Table 1 resumes the evolution of weights in each iteration. 


\begin{tabular}{|c|c|c|c|}
\hline \hline Iteration & Expert 1 & Expert 2 & Expert 3 \\
\hline \hline 0 & 1 & 0 & 0 \\
\hline 1 & 0.368809216192937 & 0.297426787252369 & 0.333763996554694 \\
\hline 2 & 0.337704855120950 & 0.321717143517176 & 0.340578001361874 \\
\hline 3 & 0.336249576125929 & 0.323795310037380 & 0.339955113836691 \\
\hline 4 & 0.336159924292944 & 0.323955884376264 & 0.339884191330792 \\
\hline 5 & 0.336153654216238 & 0.323967955371357 & 0.339878390412405 \\
\hline 6 & 0.336153196429720 & 0.323968855794958 & 0.339877947775322 \\
\hline 7 & 0.336153162568463 & 0.323968922812703 & 0.339877914618833 \\
\hline
\end{tabular}

Table 1. Results of each iteration

\section{Conclusion}

In this work, we adapted Lees algorithm to achieve group consensus in the interval-valued intuitionistic fuzzy context. We restricted ourselves to the interval-valued intuitionistic fuzzy weighting averaging operator to merge opinions, used the hamming metric to compute their distances and derived similarities as a distance dual. In future research, we will investigate different combinations of aggregation operators, similarities and distances that may be more appropriate in such situations.

\section{References}

[1] Atanassov, K. (1983) Intuitionistic fuzzy sets, VII ITKR Session, Sofia, 20-23 June 1983 (Deposed in Centr. Sci.-Techn. Library of the Bulg. Acad. of Sci., 1697/84) (in Bulgarian). Reprinted: Int. J. Bioautomation, 2016, 20(S1), S1-S6.

[2] Atanassov, K. (1986) Intuitionistic fuzzy sets, Fuzzy Sets and Systems, 20, 87-96.

[3] Atanassov, K. (1999) Intuitionistic Fuzzy Sets: Theory and Applications, Physica-Verlag Heidelberg.

[4] Atanassov, K., \& Gargov, G. (1989) Interval valued intuitionistic fuzzy sets, Fuzzy Sets and Systems, 31, 343-349.

[5] Hsu, H. M., \& Chen, C. T. (1996) Aggregation of fuzzy opinions under group decision making, Fuzzy Sets and Systems, 79, 279285.

[6] Koczy, T. L., \& Tikk, D. (2000) Fuzzy rendszerek, Budapest.

[7] Lee, H. S. (2002) Optimal consensus of fuzzy opinions under group decision making environment, Fuzzy Sets and Systems, 132, 303-15.

[8] Lu, C., Lan, J., \& Wang, Z. (2006) Aggregation of Fuzzy Opinions Under Group DecisionMaking Based on Similarity and Distance, J. Syst. Sci. Complex., 19, 63-71. 
[9] Santini, S., \& Jain, R. (1997) Similarity is a Geometer, Multimed. Tools Appl, 5, 277-306.

[10] Williams, J., \& Steele, N. (2002) Difference, distance and similarity as a basis for fuzzy decision support based on prototypical decision classes, Fuzzy Sets and Systems, 131, 3546.

[11] Xu, Z., \& Cai X. (2012) Intuitionistic Fuzzy Information Aggregation: Theory and Applications, Springer-Verlag, Berlin Heidelberg.

[12] Yue, Z. (2011) Deriving decision makers weights based on distance measure for intervalvalued intuitionistic fuzzy group decision making, Expert Syst. Appl. 38 (9), 11665-11670.

[13] Zadeh, L. A. (1965) Fuzzy sets, Information and Control, 8, 338-53.

[14] Zhang, X., \& Xu, Z. (2015) Soft computing based on maximizing consensus and fuzzy TOPSIS approach to interval-valued intuitionistic fuzzy group decision making, Appl. Soft Comput., 26, 42-56. 